\section{J. BOUGLER}

Institut National Agronomique Paris-Grignon, Département des Sciences Animales

16 rue Claude Bernard 75231 Paris Cedex 05
La gestion des populations

\section{La loi sur l'élevage et l'organisation générale de la sélection en France}

Résumé. Pour obtenir de bons résultats en amélioration génétique, il faut des objectifs clairs, des méthodes éprouvées, des hommes compétents et une organisation assurant la cohérence des actions entreprises par chacun des partenaires. La Loi sur l'Elevage mise en place en France en 1966 a apporté aux élevages français le cadre correspondant qui est ici brièvement décrit. Le Département de Génétique Animale de l'INRA a, depuis le début, joué un rôle essentiel pour concevoir, mettre en place et assurer le suivi de toute cette organisation.

L'élevage français occupe, dans le concert mondial, une place de choix, tant par l'importance et les niveaux de production de ses cheptels des diverses espèces que par l'originalité et l'intérêt de beaucoup de ses races. Cette place, nous la devons à notre histoire, à notre géographie, à la qualité de nos éleveurs, mais aussi à notre organisation de sélection qui s'est trouvée largement perfectionnée, il y a un quart de siècle, à la suite de la Loi sur l'Elevage du 28 décembre 1966.

Il fallait en effet, à cette époque, adapter notre appareil de sélection progressivement mis en place au cours des décennies précédentes (Livres généalogiques : 1860-1920, contrôle des performances : 19101950, insémination artificielle : 1950-1970, organisation économique de la production : $1960 \ldots$.., à l'arrivée de nouvelles connaissances et de nouvelles technologies (la génétique quantitative, le calcul automatique, le développement de l'IA), afin de lui permettre d'atteindre à une plus grande efficacité. L'apport des chercheurs généticiens et le travail de réflexion conduit en commun avec les responsables publics (Ministère de l'Agriculture) et professionnels furent alors déterminants : ils ont permis de fixer le cadre dans lequel s'est depuis développée l'organisation génétique de l'élevage français.

\section{1 / Les espèces concernées}

L'aviculture, secteur où les activités de production sont bien structurées au sein de filières et où les entreprises de sélection sont en nombre très limité, ne fait pas l'objet d'une quelconque réglementation génétique ; les lois du marché, l'organisation économique et la concurrence entre entreprises sont les éléments moteurs et régulateurs de ce secteur.

Le cheval, de son côté, était dans des conditions particulières puisqu'il bénéficiait de l'encadrement du Service des Haras, traditionnellement en charge des actions d'amélioration génétique des races, ainsi que d'un financement particulier (prélèvement sur les courses) ; il n'a ainsi été rattaché aux dispositions découlant de la Loi sur l'Elevage de 1966 que deux ans plus tard, et seulement pour certaines actions visant notamment l'identification et les enregistrements zootechniques, le fonctionnement des Livres Généalogiques et quelques opérations de sélection (telle par exemple l'insémination artificielle).

Les autres espèces (bovins, ovins, caprins, porcins) tombaient par contre totalement dans le champ d'application de la Loi sur l'Elevage, dont beaucoup de dispositions prises en application ont souvent à l'origine été inspirées par la situation relative aux bovins laitiers (l'insémination artificielle s'y étant développée plus tôt et y étant plus répandue, c'est ce secteur qui, en 1966, marquait le plus la rupture par rapport à la situation antérieure).

\section{2 / A la base, un seul interlocuteur et des méthodes standardisées}

La mise en place, dans chaque département, d'un Etablissement de l'Elevage (EDE) rassemblant les diverses structures préexistantes ou travaillant en contrat avec elles a permis de disposer, sinon d'un interlocuteur unique, du moins d'une étroite coordination pour toutes les opérations de base du travail de sélection, à savoir l'identification, l'établissement des filiations et le contrôle des performances. Ces opérations demandent en effet à être réalisées par des agents proches des élevages (bonne connaissance des troupeaux, correction rapide des erreurs, réduction des coûts,...) et selon une méthodologie commune (numéro unique par animal, méthodologie bien standardisée pour les contrôles).

L'homogénéité de méthodologie d'un bout à l'autre du territoire national est réalisée grâce à la mise en oeuvre de règlements techniques pour chaque opéra- 
tion de contrôle : ces règlements, préparés par les organismes de recherche (INRA) en liaison avec les partenaires professionnels (Instituts Techniques d'Elevage), sont agréés par le Ministère de l'Agriculture et communiqués aux organismes de terrain chargés de leur mise en oeuvre ; le suivi de leur application, en général encouragé par des aides en provenance du Ministère de l'Agriculture ou des Offices, est alors réalisé par l'Institut Technique de l'espèce correspondante.

\section{3 / Un encouragement au développement des bases de sélection}

Dans les méthodes classiques de "sélection ouverte" (élevage en race pure, évaluation des reproducteurs en ferme, échanges de géniteurs entre élevages), on a intérêt à disposer d'un nombre élevé d'animaux à la fois identifiés, filiés et contrôlés (base de sélection); il est aussi important, tant pour diffuser le progrès génétique, réaliser des accouplements raisonnés ou mieux connecter les élevages entre eux en vue d'améliorer la précision des évaluations génétiques, qu'une fraction aussi élevée que possible des femelles correspondantes soit inséminée (population active).

Des progrès importants ont été réalisés en ce domaine depuis les années 70 (tableau 1), qui tiennent tant à l'organisation mise en place et à l'évolution générale des pratiques des éleveurs qu'aux aides financières qui ont été accordées pour faciliter le développement de ces contrôles ou à l'apparition de nouvelles techniques (ultrasons, pesée automatique,...).

Tableau 1. Evolution des bases de sélection et des effectifs de femelles inséminées de 1970 à 1990 .

\begin{tabular}{|c|c|c|c|c|c|}
\hline Effectifs contrôlés & 1970 & 1975 & 1980 & 1985 & 1990 \\
\hline vaches laitières & 1060000 & 1548000 & 2025000 & 2408000 & 2605000 \\
\hline vaches allaitantes & & 114000 & 134000 & 165500 & 250000 \\
\hline brebis laitières & 36000 & 87000 & 118000 & 156000 & 213000 \\
\hline brebis allaitantes & 138500 & 386000 & 404000 & 395000 & 405000 \\
\hline F8 & & 168000 & 168000 & 153000 & 113000 \\
\hline F1 & & 131000 & 126000 & 143000 & 199000 \\
\hline $\mathrm{F} 2$ & & 87000 & 110000 & 98000 & 92000 \\
\hline chèvres laitières & 24000 & 48000 & 100000 & 114000 & 250000 \\
\hline truies en sélection & 6700 & 15500 & 20900 & 19700 & 15000 \\
\hline Femelles inséminées & 1970 & 1975 & 1980 & 1985 & 1990 \\
\hline vaches & 7328000 & 7158000 & 7042000 & 6602000 & 5774000 \\
\hline dont - laitières & - & - & - & 6155000 & 5296000 \\
\hline - allaitantes & - & - & - & 447000 & 478000 \\
\hline brebis & 20000 & 94000 & 249000 & 398000 & 607000 \\
\hline - laitières en IA & & & (14\%) & & $(29 \%)$ \\
\hline - allaitantes en IA & & & $(1 \%)$ & & $14 \%)$ \\
\hline chèvres & & 4200 & 8600 & 17000 & 51000 \\
\hline truies & 24000 & 50000 & 70000 & 95000 & 135000 \\
\hline
\end{tabular}

\section{4 / Un réseau national de collecte et de traitement de l'information}

Le développement des contrôles ne peut se réaliser que si les informations élaborées que l'on en tire reviennent rapidement à l'éleveur pour l'aider dans la gestion de son élevage, et justifier ainsi le versement de sa participation aux frais de contrôle ; parallèlement, il n'est utile à l'amélioration génétique que si les informations de base convergent toutes vers le Centre informatique national chargé de la réalisation des calculs conduisant à l'évaluation génétique, en l'occurrence le CTIG de Jouy-en-Josas.

C'est pour atteindre ce double objectif que le réseau national de collecte et de traitement des informations zootechniques a été structuré de la manière suivante :

- à la base, les EDE, chargés de vérifier les informations adressées par les éleveurs et de collecter, puis de vérifier, celles recueillies par des techniciens

- au niveau régional, des Centres Régionaux Informatiques (CRI) qui traitent les informations correspondantes aux fins de gestion, en vue d'un retour rapide des résultats correspondants auprès des éleveurs, et envoient les données de base vers l'atelier national. Dix CRI existent en France, chacun étant engagé dans une ou plusieurs chaînes informatiques (bovins laitiers, bovins allaitants, ovins laitiers,...). Chaque CRI gère le fichier régional et est l'interlocuteur de l'échelon national pour les animaux correspondants

- au niveau national, toutes les informations en provenance des CRI et, pour les pointages, des UPRA et organismes associés, sont gérées par le CTIG de Jouy-en-Josas (fichier national). C'est cet atelier qui calcule tous les index et envoie les informations élaborées vers, d'une part, les fichiers régionaux (CRI), d'autre part, les fichiers raciaux gérés pour chaque race par l'UPRA (ces fichiers raciaux sont, en pratique, souvent traités sur le site du CTIG ou d'un CRI ; chez les ovins, le fichier géré par le CTIG a la double fonction de "national" et de "racial" = fichier "national-racial").

\section{5 / Pour chaque race, un pilote : l'UPRA}

La Loi sur l'Elevage de 1966, en même temps qu'elle confiait la tenue des filiations aux EDE, rénovait en profondeur les Livres Généalogiques en suscitant leur transformation en Unités Nationales de Sélection et de Promotion de race (UPRA) : les anciens Livres Généalogiques s'ouvraient à tous les éleveurs de la base de sélection, aux autres Unités de Sélection conduisant des programmes de sélection de reproducteurs mâles (Centres de Production de semence) et aux utilisateurs de la race et de ses produits. Cette vaste ouverture était indispensable pour constituer, dans chaque race, une véritable UPRA chargée d'assumer le destin de cette race, c'est-à-dire d'en définir et d'en organiser le programme de sélection et de promotion : choix des objectifs de sélection, choix des critères à contrôler, définition de la grille de qualification, coordination des procédures de sélection, mise en oeuvre de la politique de promotion...

Aujourd'hui, la quasi-totalité des races possèdent une structure d'UPRA mais force est de constater que, dans beaucoup de situations, ces UPRA ne fonctionnent pas encore tel qu'on pourrait le souhaiter : 
représentation trop faible des utilisateurs, mauvaise intégration des représentants des $2^{\circ}$ et $3^{\circ}$ Collèges, encadrement technique trop limité, interférences entre les aspects techniques et les aspects commerciaux... Ces difficultés tiennent à plusieurs causes : au fait tout d'abord que le financement des UPRA est principalement assuré, hors subventions, par les éleveurs du $1^{\circ}$ Collège, en échange de services rendus ; au fait ensuite que les divers participants, à défaut d'entente sur leurs missions respectives, conduisent parfois des actions insuffisamment concertées ou même concurrentes ; et peut-être enfin au poids des habitudes passées des uns et des autres! On peut toutefois espérer que ces difficultés vont s'estomper rapidement et que, grâce aux efforts de tous, de véritables UPRA, des UPRA de filière, se développeront dans chaque race pour assurer le nécessaire travail d'orientation et de coordination des efforts de chacun.

\section{6 / La maîtrise de l'outil IA}

Si la monte privée est en principe libre, tout rapprochement entre reproducteurs ou gamètes habituellement entretenus en des lieux différents (monte publique) fait l'objet d'une réglementation dont l'objet est de s'assurer que, tant au plan zootechnique que sanitaire, les actions correspondantes concourrent à la création ou à la diffusion d'un progrès. Dans le cas de l'IA, mode de reproduction largement utilisé dans quelques productions (bovins et ovins laitiers) et que l'on s'efforce de développer dans les autres, la réglementation porte ainsi sur les organismes, sur les personnes et sur les animaux eux-mêmes : l'agrément accordé à chacun de ces niveaux permet de s'assurer que toutes les conditions sont réunies pour que le travail soit efficace et de qualité.

Les conditions correspondantes, édictées depuis le début des années soixante-dix et régulièrement remises à jour depuis, sont actuellement revues de façon à être harmonisées avec les dispostions qui sont en train de se mettre en place au niveau de la CEE.

\section{7 / L'encadrement national du dispositif}

Toute cette organisation demande à être pilotée, pour à la fois s'assurer qu'elle fonctionne selon les règles prévues et lui permettre toutes les évolutions souhaitables. Ce pilotage est assuré par un double système :
- d'une part, au moyen d'un encadrement national : ce sont les équipes Amélioration Génétique de chaque Institut ou des organisations de même nature, lesquelles travaillent en étroite liaison avec les généticiens de l'INRA et les responsables du Ministère de l'Agriculture. Il est ainsi possible, en utilisant la voie descendante, de proposer aux organismes de base des méthodes de travail intégrant les derniers progrès des connaissances ou des techniques (l'exemple de l'indexation est très illustratif); de même, par la voie ascendante, tout problème de terrain peut être étudié par les spécialistes et ainsi des solutions être apportées. L'utilisation du réseau informatique, et les tableaux de bord qui maintenant existent pour chaque chaîne de traitement, sont aussi des outils essentiels pour assurer ce pilotage quotidien des opérations de sélection.

- d'autre part, par le biais de l'agrément des organismes et des méthodes et le financement des actions correspondantes (chapitre budgétaire 44-50, budget des Offices,...). Ce rôle revient naturellement au Ministère de l'Agriculture, éclairé pour les décisions correspondantes par les Comités consultatifs d'espèce de la Commission Nationale d'Amélioration Génétique (CNAG), lesquels sont composés de représentants de l'Administration (Ministère de l'Agriculture, INRA, Enseignement supérieur) et de la Profession (chaque famille professionnelle concernée y est représentée).

Il est ainsi possible d'assurer l'animation et l'orientation de tout le dispositif national d'amélioration génétique ainsi que, le cas échéant, de procéder à d'éventuels arbitrages.

\section{Conclusion}

L'amélioration génétique est une oeuvre collective qui, aujourd'hui, compte tenu de l'organisation de notre élevage, fait intervenir de nombreux partenaires dont il importe de coordonner les actes. Le développement d'entreprises dans ce secteur constitue toutefois une caractéristique nouvelle qui, avec l'irruption des nouvelles technologies et l'ouverture des frontières, deviendra de plus en plus importante ; l'organisation actuelle sera donc conduite à évoluer, et c'est ce qu'elle a déjà commencé à faire comme on peut le constater lorsque l'on examine la diversité des situations selon les espèces et les productions. 